\section{DNA SYNTHESIS}

Priming the Polymerase

from our Cell Biology Correspondent

Playing David is a risky business, for most giants are capable of putting up a deal more resistance than Goliath managed and either drive their challengers from the field or, what is perhaps even more humiliating, drive them to oblivion simply by not deigning to take notice. But, irrespective of the final outcome, few people are not enthralled by the sight of somebody trespassing on some giant's backyard only to throw a stone through his window. One cannot help wondering, for example, what will be the response to Keller's report, in the current issue of the Proceedings of the National Academy of Sciences $(69,1560 ; 1972)$, of experiments which prove that RNA primer molecules can initiate the synthesis of phage $\phi \times 174$ DNA by mammalian as well as bacterial DNA polymerase.

The notion that the initiation of bacterial and phage DNA synthesis might be effected by an RNA primer molecule rather than a DNA primer stems from the observation, made by several groups, that the replication of Escherichia coli DNA and some coliphage DNAs seems to be tightly linked to the transcription of the DNA genome which is to be replicated. When transcription is prevented by one means or another DNA replication is inhibited.

One of the most intriguing interpretations of this finding, which was pointed out by Kornberg and his associates last year, is that transcription might be required because it might provide a short RNA chain, which, complementary to its template DNA strand, could act as a primer presenting a free 3' hydroxyl group onto which DNA polymerase could polymerize deoxynucleoside triphosphates. Not so many years ago such an unorthodox idea might well have been killed by ridicule had it ever appeared in print. But the discovery that RNA tumour virus particles contain an enzyme which is capable of using RNA molecules both as primers and as templates for the synthesis of complementary DNA so changed the climate of opinion that by last year the suggestion that an RNA might prime DNA replication seemed not at all unreasonable. Indeed it has now been verified unambiguously by Keller's elegant experiments with appropriate in vitro systems.

Keller found that if DNA polymerase II extracted from KB cells, which are of human origin, or if DNA polymerase I from the bacterium Micrococcus luteus is offered pure coliphage $\phi \times 174$ DNA (single stranded and covalently circular molecules) as template, polymerization of deoxynucleoside triphosphates into complementary DNA does not occur. When, however, DNA dependent RNA polymerase from $E$. coli, together with the four ribonucleoside triphosphates, are added, RNA and DNA synthesis begins and any interference in RNA synthesis results in a diminution of DNA synthesis.

In this most heterologous cell free system DNA synthesis could, however, be uncoupled from RNA synthesis if the DNA polymerases were offered as template DNA/RNA hybrids produced by preincubating the DNA with the RNA polymerase and its substrates. Reverse transcriptase from avian myeloblastosis virus particles could not substitute for DNA polymerase in either the coupled or uncoupled reactions. All these data suggest strongly that the DNA synthesis is being primed by an RNA primer, and Keller confirmed that the newly made DNA is linked to RNA by a $3^{\prime}: 5^{\prime}$ phosphodiester bond by physical and chemical tests. He then proceeded to show that the product of the coupled RNA-DNA synthesis reaction is a double stranded nucleic acid comprising the template DNA chain hydrogen bonded to the product RNA-DNA chain by exposing the product to ribonuclease $\mathrm{H}$ from $\mathrm{KB}$ cells, which specifically digests the RNA moiety of RNA-DNA hybrids, and to Neurospora nuclease which degrades single but not double stranded RNA or DNA.

These experiments clearly prove that RNA chains can prime the initiation of DNA synthesis. To what extent prokaryotic and eukaryotic organisms avail themselves of this potential has yet to be determined, but as Keller points out the widespread distribution of RNAase $\mathbf{H}$ in eukaryotes and its occurrence in RNA tumour viruses suggest that RNA-DNA hybrid molecules may often turn up in animal cells and in bacteria RNAase III might play the part of RNAase $H$. Clearly, Keller has struck a rich seam; it will be interesting to see who joins in the rush to exploit it and who ends up as chief proprietor.

\section{Why Venus is Cloudy}

IN next Monday's Nature Physical Science (July 3) J. T. Bartlett and G. E. Hunt put forward a suggestion to account for the difference in the degree of cloud cover in the atmospheres of the Earth and Venus. They suggest that, contrary to the suggestion of Goody and Robinson (Astrophys. J., 146, 339 ; 1966), there may be no cloud free region at all over the surface of Venus.

Goody and Robinson's work depended on the assumption that a general upward motion in the atmosphere of Venus produced widespread cloud cover, just as cloudy regions on Earth are generally associated with areas of upward motion in the atmosphere; but these authors went on to conclude that this general upward movement must be countered by a small region of descending atmosphere on the cooler (dark) side of Venus. Such a cloud free region could, they argued, be too small to detect from Earth using present observational equipment.

On the basis of more recent evidence, including the direct measurements made by the Venera 7 probe, Bartlett and Hunt cescribe a two component model of the atmosphere in which a dense cloud layer in the troposphere covers a diffuse haze in the stratosphere.

It seems that such a model is consistent with the presence of a supersaturation of the cloud forming substance-whatever that may be-in the Venus atmosphere. Because of the decrease of pressure and temperature in the troposphere, this substance might exist as a vapour in the lower regions of the atmosphere but there will be a region completely enclosing the planet in which the atmosphere is supersaturated with the cloud forming substance. Irregular mixing of the various components of the atmosphere will produce irregularities in the cloud bases; but a continuous cloud cover will exist, say Bartlett and Hunt, provided there is always some level in the troposphere above which the partial pressure of the cloud forming substance exceeds its saturation vapour pressure.

On Earth, those clouds which do form can be dispersed either because of precipitation or because they become mixed with unsaturated air. But if there were no efficient precipitation mechanism, as seems to be the case on Venus, the available pool of cloud forming material on the planet would continue to evaporate until the air in contact became saturated. Of the two precipitation mechanisms operating on Earth one depends on the coalescence of water droplets and the other on the presence of supercooled liquid together with a few solid particles which act as seeds.

According to Bartlett and Hunt, there is no reason to believe either that an appropriate distribution of droplet sizes exists on Venus for the first mechanism to operate, or that the cloud forming substance-whatever it may be-exists within the critical temperature range close to its freezing point necessary for the second mechanism to work.

The cloud forming process on Venus, it seems, is fundamentally different from that of Earth, where an efficient rain making mechanism ensures only partial cloud cover. 